\title{
La justicia social en el análisis de las políticas educativas: una mirada desde la obra de Stephen Ball
}

\author{
Social justice in the analysis of educational \\ policies: a look from the work of Stephen Ball
}

\author{
Debie Buelto Harry* \\ debieharry@hotmail.com \\ Orlando David Murillo* \\ omurillo86@gmail.com \\ Zuleyma Isabel Peña* \\ argueta_zuleyma@yahoo.com \\ Cecilia Alejandra Mejía ${ }^{11}$ \\ ceciliamejiamejia@gmail.com
}

\section{Resumen}

Este artículo analiza la La noción de justicia social en los perspectiva de Stephen Ball sobre justicia social en el estudio de las escritos de Ball sobre políticas educativas se caracteriza por la políticas educativas relacionadas con micro-política, elección de la escuela por los padres, neoliberalismo, papel del Estado, filantropía, reformas educativas, su impacto en la vida escolar y su constante reinterpretación por los diferentes actores educativos. búsqueda de mecanismos que permitan empoderar a un educando, una institución o región para que pueda alcanzar un nivel aceptable de desarrollo, su mirada está identificada con una amplia visión sobre los problemas de igualdad y equidad social en

11* Estudiantes de la I Promoción del Doctorado Latinoamericano en Políticas Públicas y Formación Docente, UPNFM.

Recibido 1 de septiembre 2017 / Aceptado 25 octubre 2017. 
La justicia social en el análisis de las políticas educativas: una mirada desde la obra de Stephen Ball

la escuela y su contexto social, las desigualdades en el acceso escolar, la inclusión social de los sectores históricamente marginados y la reducción de brechas sociales, entre otros. Igualmente se traza el cambio de la idea de educación como valor público a uno centrado en el mercado, referido por Stephen Ball como excluyente, al caracterizarle estrategias que privatizan la educación pública.

Palabras clave: política educativa, justicia social, el Estado, neoliberalismo y reforma educativa.

Abstract

This article analyzes Stephen Ball's perspective on social justice in the study of educational policies related to: micro-politics, choice of school by parents, neoliberalism, role of the state, philanthropy, educational reforms, its impact on school life and its constant interpretation by the different educational actors.

The notion of social justice in Ball's writings on educational policies is characterized by the search for mechanisms that allow empowering an educator, an institution or region so that it can reach an acceptable level of development, its vision is identified with a broad vision on the problems of equality and social equity in the school and its social context, the inequalities in school access, the social inclusion of historically marginalized sectors and the reduction of social gaps, among others.

It also traces the change of the idea of education as a public value to one centered on the market, referred to by Stephen Ball as excluding, by characterizing strategies that privatize public education.

Keywords: educational policy, social justice, the State, neoliberalism and educational reform.

<Paradigma> - Revista de Investigación Educativa. Año 24. No. 38 


\section{Introducción}

Las políticas educativas y políticas en general son acciones ligadas al desarrollo social de un país, región, comunidad y/o institución, aunque desde diversas perspectivas suelen coincidir en que transversalmente están relacionadas por el tema de justicia social, de manera tal que para tener avances implica que "Ileguemos todos, que no se excluya, que se integre a los excluidos" (Puigros, 2001)

Esta idea de incorporar en las políticas educativas la concepción de justicia social ha motivado a Stephen Ball ${ }^{12}$-profesor de Sociología de la Educación en el Instituto de Educación de la Universidad de Londres, quien investiga y publica sobre temáticas de teoría de la organización escolar, etnografía, mercados educativos, micropolítica, reformas educativas, privatización de la educación, la filantropía como posible forma de privatización, la elección de la escuela por los padres, cambios en el discurso educativo y escolar, performatividad, impacto de las reformas en el trabajo y la identidad de los profesores y demás profesionales que interactúan en educación y redes políticas. Sus escritos han recibido la contribución de pensadores como Foucault, Bourdieu, Bernstein, Weber, entre otros, (Mainardes., \& Marcondes, 2009, pp. 303-304).

En este artículo proponemos desde un posicionamiento crítico analítico estudiar la noción de justicia social en las políticas educativas valorando la obra de Stephen Ball, he indagando en algunos conceptos como capital cultural, reproducción de desigualdades, modelo económico, relaciones de poder, y la incidencia de estos factores en el ciclo de las políticas vistas como texto o discurso.

Para realizar este análisis, metodológicamente se utilizaran las tres dimensiones que Nancy Fraser (2009, p. 17) propone: 1. Dimensión económica de la distribución (reclamación por la redistribución y

${ }_{12}$ La mayoría de sus publicaciones son en el idioma inglés; para la elaboración de este artículo se utilizaron traducciones libres de sus textos a la lengua española. 
La justicia social en el análisis de las políticas educativas: una mirada desde la obra de Stephen Ball

afirmación económica). 2. Dimensión cultural del reconocimiento. 3. Dimensión política de la representación (inclusión en la comunidad política para expresar sus demandas).

\section{La justicia social en la dinámica del poder}

Una política educativa con enfoque de justicia social deberá proporcionarle a la población legitimidad social y beneficios sociales. Esta concepción de justicia social es considerablemente inclusiva, "no especifica una raza, clase, discapacidad o sexualidad; abarca una amplia visión de los problemas de equidad, oportunidad y de justicia. Es flexible y tiene una gama considerable de aplicaciones" (Mainardes., \& Marcondes, 2009, p. 307).

Abordar el concepto de justicia social en la obra de Stephen Ball parece elemental y básico, significa igualdad de oportunidades, acceso igualitario, promoción de la inclusión social y la disminución de las brechas sociales, una escuela "que mantiene a todos los alumnos trabajando, comprometidos y con la sensación de que no fracasarán" (Ball, 2008). Esta idea de justicia social implica la no exclusión de los individuos, en donde el éxito de un sistema está en abrir las posibilidades y oportunidades a la humanidad y procurar que lleguen todos.

La postergación de los grupos más vulnerados y vulnerables en nuestros contextos sociales y culturales han respondido históricamente a una dinámica de poder que reproduce una sociedad que no garantiza el acceso igualitario al capital cultural. Esto sucede en América Latina con las mujeres, el mundo rural, las poblaciones indígenas y con los que no tienen los recursos económicos para comprar servicios educativos de calidad, pero no es solamente un drama endémico de esta región, pasa también con los inmigrantes en Europa y con aquellos ciudadanos de los países desarrollados que solo pueden elegir escuelas y universidades que se apeguen a sus limitaciones económicas, quedando por fuera de un mercado que reserva los empleos con mayor remuneración para los más calificados, entendiendo por ello, los 
graduados de instituciones posicionadas en los índices de rendimiento y calidad, cerrando el ciclo y perpetuando la reproducción de un sistema credencialista (Collins, 1989).

El término de justicia social pone en cuestión la noción de clases sociales, en donde se evidencia relaciones de poder y que sugiere lo que Foucault (2002) denominó como "poder no localizado", es decir, un conjunto que invade todas las relaciones sociales y que se manifiesta no solo en las políticas sino también en las aspectos tan cotidianos como la lengua, donde los éxitos de los actos del habla depende en gran medida de la posición social y cultural del emisor con respecto al que escucha.

Sin embargo, la clase social no está definida solamente por el lugar que ocupan los individuos en las relaciones de producción, sino también lo que Bourdieu\&Passeron (1996) denominan "casualidad estructural de una red de factores", identificando otros indicadores tales como profesión, ingresos, nivel de instrucción, costumbres, modos y estilos de vida; que no siempre son producto de una elección.

En la búsqueda de la igualdad social en educación: la dimensión cultural del reconocimiento

Las desigualdades obedecen a decisiones políticas. Son el resultado de determinaciones colectivas sobre quién debe ser educado, cómo y a qué costo, "la pobreza es el resultado de una elección, pero no de los pobres, sino de los no pobres. Es el resultado de cómo los no pobres definen sus intereses y responsabilidades y cómo influyen en las prioridades de la acción pública" (Reimers, 1999).

La búsqueda de la igualdad social en la educación parece más bien una quimera en un sistema diseñado para profundizar las diferencias sociales convirtiendo la carrera por la educación en una lucha simbólica por el reconocimiento y por el capital cultural. Al respecto Ball (2010) sustenta que la justicia y la igualdad social no solo debe buscarse con reformas en los centros educativos, "además es una cuestión de valores cívicos, sociabilidad y liderazgo político". 
La justicia social en el análisis de las políticas educativas: una mirada desde la obra de Stephen Ball

La dimensión cultural de reconocimiento de Nancy Fraser puede ser entendida como la necesidad social de "visibilización del otro", la aceptación de una realidad social y cultural en la que se reclama espacios de participación por los diferentes sectores sociales para la toma de decisiones, rediseñando las políticas sociales, ampliando los sistemas de co-gobernaza, y considerando en la mesa de debate aquellas demandas sociales de quienes históricamente les ha costado más ser escuchados y tomados en cuenta.

Plasmar esta dimensión en el terreno educativo pasa por analizar el ciclo de las políticas y revisar la trayectoria que siguen las demandas sociales para llegar a la mesa de discusión donde estas se diseñan como texto, y posteriormente, analizar el impacto que tienen en la vida escolar y cómo son reinterpretadas por los actores educativos convirtiéndolas en lo que Ball llama "políticas como discurso", estudiar todo este recorrido requiere de una investigación más robusta por lo que no se pretende en este trabajo profundizar en este aspecto.

Lo que se puede inferir a partir del análisis de los aportes de Ball es que existe una correlación entre el rendimiento escolar de los educandos y el contexto socioeconómico, un ejemplo claro es el logro educativo medido en las pruebas internacionales que en su mayoría es más alto en los países desarrollados. Los resultados de las evaluaciones escolares están incidiendo en la aplicación de prácticas por parte de los centros educativos en la selección de sus estudiantes "moduladas por hipótesis con respecto a las intersecciones de clase, raza, origen étnico y género" (Ball., \& Youdell, 2007, p. 44).

En esta búsqueda de la igualdad social en educación, los países de América Latina y el mundo han optado por la implementación de políticas educativas que han dado como resultado la ampliación de los servicios educativos, aumentando el porcentaje de niños que asisten a las escuelas y que posteriormente aspiran a estudios superiores, paralelo a ello, también crecen las diferencias entre los grupos socioeconómicos más favorecidos y los menos favorecidos, así como entre los que son dueños del capital simbólico como la lengua oficial 
y la cultura dominante; y los grupos de minorías étnicas, religiosas, sexuales, económicas, lingüísticas y que buscan dentro de su dimensión cultural el reconocimiento.

El neoliberalismo como motor de la privatización y gestor de las desigualdades: la dimensión económica de la distribución

Es claro que las nuevas formas de gobernanza apelan por cada vez menos concentración de los mecanismos de poder, sugiriendo nuevas formas de co-gobernanza e involucrando al menos en teoría a todos los actores sociales para incidir en la toma de decisiones que se convertirán en las nuevas políticas económicas, sociales o culturales. Entender la dimensión económica de la distribución que propone Fraser \& Honneth (2003) significa pasar una rápida mirada por el modelo económico que impera en la región, el neoliberalismo en su sentido más práctico propone una desregulación del papel del Estado como proveedor de servicios públicos y concibe estos últimos como tarea de asociaciones o instituciones que tienen como finalidad primordial el lucro.

Ball (2016) argumenta que al mismo tiempo que el Estado cumple con el rol de establecer límites para los actores del mercado (las empresas, la filantropía, etc.) crea las condiciones para que el mercado florezca y se expanda. Es decir, que el desplazamiento del gobierno a la gobernanza se basa en una forma distinta de ejercer el poder y la autoridad en la cual hay una división del trabajo entre el Estado y otros actores gubernamentales, no gubernamentales, con fines de lucro, sin fines de lucro, globales y locales que participan en la toma de decisiones, de la puesta en acto de las políticas, de la provisión de servicios educativos y de la evaluación de las propias políticas y sus resultados (Beech \& Meo, 2016, p. 7).

Esta forma de concebir el Estado y sus funciones es una sugerencia de las grandes instituciones bancarias que gestionan y administran la deuda de los países de la región como parte de un modelo económico pensado para promover la inversión del capital privado y generar 
La justicia social en el análisis de las políticas educativas: una mirada desde la obra de Stephen Ball

desarrollo desde el ámbito empresarial. Las promesas de desarrollo social del neoliberalismo así como la teoría del capital humano fueron meras quimeras que únicamente profundizaron las desigualdades sociales al dejar de lado la inversión y mejora de los servicios públicos que paulatinamente fueron adquiriendo costos muy elevados en nombre de una mejor calidad.

Esta ola neoliberal impulsó modelos de descentralización para disminuir la burocracia estatal y eficientar los procesos de toma de decisiones, arquetipos que a lo sumo alcanzaron una desconcentración cuya tutela del Estado se ha mantenido vigente salvo algunos casos de cuasi mercados que en un periodo de tiempo parecían dar resultado en algunos países de América Latina, pero que poco a poco se fueron convirtiendo en aparatos de exclusión (Fischman., Ball., \&Gvirtz, 2003, p. 2).

Aplicando a esto un análisis basado en el ciclo de políticas públicas propuesto por Stephen Ball es preciso revisar los espacios donde las micropolíticas transforman las vidas cotidianas y donde los resultados son tan múltiples y variados que clasificarlos en buenos y malos implicaría un mero simplismo. Para no caer en la tentación de juzgar por los efectos encausaremos este análisis crítico en uno de los aspectos que más nos interesa: la educación y cómo entender el neoliberalismo como motor de desigualdades.

Esto no debe sugerir que las desigualdades surgen única y exclusivamente con este modelo económico, pero sí se profundizan con él, partiendo de una idea globalizadora que no pensó en globalizar las oportunidades, ni el acceso, ni la calidad de la educación para aquellos que ya venían arropados por la exclusión y la pobreza. La efervescencia por la privatización como la solución a la incapacidad del Estado para suplir las demandas sociales fue una de las causas principales para impulsar diferentes modelos privatizadores en la región, convirtiendo la educación, en una lucha por acceso, por calidad, por inclusión, por reconocimiento, sumado a ello, las exigencias también válidas de los sectores que teniendo todo lo anterior, exigen mejoras. 
La desigualdad social según el ciclo de las políticas viene determinado por la trayectoria que siguen los temas que entran a la mesa de discusión donde se toman las decisiones que se convertirán en políticas. Esta selección de los temas no es una selección natural basada en las demandas o en una priorización de deudas sociales sino más bien en la influencia de los medios de comunicación masiva "quienes desempeñan un papel clave en el establecimiento de la agenda, es decir, el poder de determinar qué temas forman parte del debate" (Pearsons, 2007, p.143), aunado a ello, las exigencias de los organismos financieros internacionales y en la mercantilización de las capacidades poblacionales donde América Latina se perfila como mano de obra para las grandes industrias transnacionales de los países que son potencias mundiales.

Ante esta panorámica Stephen Ball plantea un análisis basado en el estudio del ciclo que conlleva estas políticas, y ofrece herramientas para entender cómo operar las políticas y pensar sus resultados desde la realidad particular de cada contexto. Plantea dos formas de concebir el análisis de las políticas: como texto y como discurso. En este sentido, "conceptualizar a la política como "texto" implica entenderla como "intervenciones textuales", como codificaciones multifacéticas producidas a través de compromisos, luchas, e "interpretaciones y reinterpretaciones públicas autorizadas” (Ball, 1993, p. 11).

Aplicando el concepto de "políticas como texto" al análisis de neoliberalismo como motor de la privatización y gestor de desigualdad, encontramos en este planteamiento de Ball toda la base teórica que se materializa en los textos y que recogen la trayectoria del largo camino que siguen las políticas hasta la mesa del debate donde se genera la toma de decisiones. Esta llamada política "como texto" ingresa al micro-escenario social y transforman en el discurso cotidiano de quienes tienen la obligación de llevarlas a práctica. Este proceso no resulta fácil ya que en cada nivel los textos de políticas son interpretados, reinterpretados, y en ocasiones deconstruídos y puestos en práctica según la interpretación de quienes moldean, y que de alguna manera, también son (y quizá de manera no pensada) hacedores de políticas educativas, como es el caso de los profesores. 
La justicia social en el análisis de las políticas educativas: una mirada desde la obra de Stephen Ball

Es en estos escenarios donde se dan las luchas por el control de los discursos y donde se hacen efectivas las políticas de distribución, reconocimiento y dimensión política. Igualmente, es donde más presente está la idea de justicia social que atraviesa la obra de Stephen Ball, debido a la necesidad de que se reconozcan los mecanismos que profundizan las diferencias sociales en términos de acceso y calidad educativa, donde la desigualdad y la privatización han tomado múltiples formas y que es preciso reconocer. Tal es el caso de las organizaciones no gubernamentales (ONG's) cuya proliferación y su diversidad de funciones han convertido la filantropía en una suplente de las tareas de los Estados y en una forma de establecer diferencias entre los que acceden a la educación por méritos y selección propia, y los que tienen que ser "apadrinados" por varias ONG's dedicadas a la tarea de facilitar acceso a la educación a cambio de captar cuantiosos recursos, que por su naturaleza filantrópica es difícil calcular (Ball, 2015).

En la obra de Ball el tema de justicia social pasa por analizar la función de todos los actores involucrados en el tema educativo y cómo cada uno de ellos se transfigura ocupando escenarios cada vez más entrelazados, impidiendo diferenciar la línea que los separa o que más bien los junta. Al identificar los efectos del neoliberalismo, la desregulación del Estado y las nuevas formas de gobernanza que modifican la vida y las relaciones de los actores educativos, se podría mencionar según el ciclo de las políticas educativas, que existe una fuerte tendencia a medir rendimientos académicos en términos meramente cuantitativos, propio de un modelo económico que invierte según los resultados, que suele dejar a la filantropía tareas esenciales como la cobertura y la calidad, así como llegar a zonas de la periferia donde se construyen y se desarrollan modelos de escuelas pobres para la gente pobre.

La inclusión de las demandas sociales en la agenda de las políticas educativas: la dimensión política de la representación

Dye (2008) define la política como "lo que los gobiernos eligen hacer o no hacer", una frase corta pero que encierra lo que se puede entender 
por una práctica en la que se define los problemas que se abordarán y los que se van a ignorar.

Ball (1998) señala la existencia de apartados en las políticas educativas que simbolizaba la creciente colonización de las mismas sobre todo en los aspectos económicos, demostrando que la política del número parece primar sobre los valores educativos, esto gracias a la combinación de cinco elementos o conjuntos de influencias: a) El uso de una combinación de descentralización, objetivos e incentivos para llevar a cabo un rediseño institucional. b) La nueva economía institucional, que pretende explicar el funcionamiento de la vida social y sus diversas instituciones. c) La performatividad, la promoción de una política operativa y conmensurable. d) La teoría de la elección pública. e) La existencia de un nuevo gerencialismo, caracterizado por la inserción de las teorías y las técnicas de gestión empresarial y el culto a la excelencia en las instituciones del sector público.

La justicia social vista desde la dimensión política de representación implica pensar en los sistemas de gobernanza y co-gobernanza. Los términos "gobierno, gobernanza y gobernabilidad" están muy ligados a la vida política de un país así como al escenario internacional que busca con ellos establecer mecanismos que permitan de alguna manera empoderar las regiones del mundo para que puedan alcanzar niveles aceptables de desarrollo. La correcta definición de estos términos así como el uso práctico de los mismos puede generar el espacio adecuado para la potencialización de estos mecanismos.

Al respecto Kooiman (2000) afirma que "Frecuentemente estas interacciones están en sí mismas basadas en el reconocimiento de las (inter)dependencias. Ningún actor por sí solo, público o privado, tiene el conocimiento y la información necesaria para dar respuesta a problemas complejos, dinámicos y diversificados" (p. 61). De manera que para materializar la justicia social que apunta Stephen Ball es preciso atender a lo señalado por Fraser (2009) quien plantea que es preciso que donde se diseñan las políticas para la toma de decisiones, se encuentren claramente representados aquellos que demandan de mayor participación en las formas de co-gobernanza. 
La justicia social en el análisis de las políticas educativas: una mirada desde la obra de Stephen Ball

Convertir una demanda social en un tema de agenda de los tomadores de decisiones y diseñadores de políticas - como texto o como discurso - es algo que aun conociendo el ciclo de las políticas de Ball (2008) parece no estar lo suficientemente claro, es decir, en qué se fundamenta la elección, qué demandas sociales serán abordadas en la mesa de discusión y cuáles no. Por ello, está dimensión tiene que ver con el hecho de en qué medida los demandantes están representados entre los que deciden, de ahí que la lucha social de reconocimiento en el aspecto cultural se convierte en una lucha política por representación.

De la educación como valor público a la educación como mercancía: la política educativa en América Latina

América Latina es la región más desigual del planeta. Las brechas económicas, sociales, culturales y políticas que aparecen entre ricos y pobres, entre urbanos y rurales, entre mujeres y hombres, indígenas y mestizos, se hacen cada vez más profundas, a pesar de los esfuerzos de muchos Estados y de los visibles reclamos de los movimientos sociales y de las alertas de la academia. Esta desigualdad en la distribución del ingreso, genera contextos desfavorables para el rendimiento promedio de los estudiantes, viéndose los Estados en la necesidad de implementar estrategias de acción afirmativas que prioricen la atención en zonas rurales de los pobres y la mujer, sistemas de cuotas en la estructura formal para estudiantes desfavorecidos, y programas de educación intercultural bilingüe dirigidos a poblaciones indígenas.

Ball propone en su obra que existe un cambio en la concepción de la educación que ha sido vista como un recurso compartido de un valor muy elevado que el Estado debe a sus ciudadanos, a un producto de consumo, una especie de mercancía propiedad de personas concretas; restándole de esa manera importancia a la perspectiva de la educación como servicio público, como "un sistema abierto a todo el mundo sin discriminaciones por motivos de género, religión, cultura o clase social, gratuito, financiado por el sector público, y gestionado y evaluado conforme a los objetivos y principios establecidos democráticamente por las autoridades públicas"(Ball., \&Youdell, 2007).

<Paradigma> - Revista de Investigación Educativa. Año 24. No. 38 
La mercantilización de la educación, el afán de cuantificar los aprendizajes y el continuo deseo de alcanzar estándares impuestos por quienes pretenden suplantar los valores de la educación pública en la formación de individuos solidarios, capaces de pensar su realidad y transformarla, parece estar cambiando vertiginosamente por la evaluación con base en resultados en un claro culto al número que nos dice qué escuela presenta los mejores índices, cuáles ganarán prestigio y quiénes merecen ser apoyadas, dejando de lado todo aquello que es imposible medir cuantitativamente y que pierde valor en el mercado (Ball, 2015).

Este cambio en las reformas educativas en Latinoamérica aborta los valores propios de la educación planteados por el enfoque humanista, subestimando las dimensiones que enriquecen el plano social y suscitando únicamente al bienestar individual, a través de elementos clave que se interrelacionan entre sí como el mercado, la capacidad de gestión y la performatividad (Ball, 2007).

La privatización en la educación pública constituye una de las estrategias de acción de los organismos multinacionales, mediante la venta de los servicios educativos y la importación de métodos y prácticas basadas en el beneficio económico (Ball., \& Youdell, 2007), otorgándole a la educación un valor comercial que tiene aristas excluyentes.

Los gobiernos de los países latinoamericanos que requieren de cooperación internacional, son asesorados por estos organismos que al saber de la incapacidad que el Estado tiene de manejar la creciente demanda generada por la sociedad civil, plantean modelos privatizadores para cubrir los espacios que no abarcan las políticas educativas vigentes en el país, a través del financiamiento en las áreas de investigación, consultas, evaluaciones y nuevas propuestas que transforman las políticas educativas, desvinculando y desvaneciendo la educación como bien público. 
La justicia social en el análisis de las políticas educativas: una mirada desde la obra de Stephen Ball

\section{Consideraciones finales}

Las escuelas son micro sociedades donde las políticas como texto y como discurso penetran las realidades cotidianas y son estos escenarios que ofrecen diversas formas de complejas resistencias que ocasionan cambios en la prácticas de dichas políticas, sin embargo, el peso de un modelo económico impuesto a fuerza de capital, un sistema de evaluación que se empeña en mostrar resultados que le permitan aspirar a fondos de agencias externas, el interés creciente de los padres de familia por aspirar a "las mejores escuelas" para sus hijos, la constante preocupación de los profesores, que si bien entienden la importancia de no sucumbir a meras cifras y abogar por los valores de la educación, temen ser juzgados como profesionales incapaces producto de una evaluación de desempeño, el asedio de las ONG`s a nivel mundial que parecen haber tomado la tarea educativa como su único medio de subsistencia. Todo lo anterior, incluidas las nuevas formas de gobernanza donde las instituciones con o sin fines de lucro, empresas, agencias nacionales o internacionales inciden en el diseño de nuevas políticas que modifican la vida de la escuela y se introducen al interior de las aulas de clase en forma de texto o de discurso. Desde ahí dentro solo quedan dos caminos: replicar esas políticas o reinterpretarlas.

Es evidente que el desarrollo y creación de nuevos tipos de negocios educativos globales, son en la actualidad formas novedosas de comercio en la economía educativa, trascendiendo la división educación pública-privada, transformando así los servicios de la educación en mercancías que pueden comercializarse y de los cuales se extraen ganancias anulando en un alto porcentaje la formación ciudadana participativa y comprometida con la justicia social. De acuerdo a lo anterior, se hace evidente que en la realidad actual las políticas educativas, están desarrolladas bajo la perspectiva economista, haciéndose más evidente la injusticia social por medio de las diferencias de clase que se acentúan cada vez más, disminuyendo de esta forma las posibilidades de movilidad y ascenso social intergeneracional a través de la educación. 


\section{Referencias Bibliográfícas}

Ball, S. (1998). Big Policies/Small World: An Introduction to International Perspectives in Education Policy. Comparative Education, 34: 2, pp. $119-130$

Ball, S. (2007). Education plc. Understanding private sector participation in public sector education. London: Routledge.

Ball, S. (2007). Reformar escuelas/ reformar profesores y los terrores de la performatividad. Revista Portuguesa de Educación, año/vol. 15, número 002, pp.1-8

Ball, S. (2008). El sentido de las reformas educativas, Santiago de Compostela: Universidad Pública. Recuperado de http://firgoa.usc.es/ drupal/node $/ 38483$

Ball, S. (2010). New Class Inequalities in Education: Why Education Policy May Be Looking in the Wrong Place! Education Policy, Civil Society and... International Journal of Sociology and Social Policy.

Ball, S. (2014). Globalización, mercantilización y privatización: tendencias internacionales en Educación y Política Educativa. Archivos Analíticos de Políticas Educativas.

Ball, S. (2015). Educación Pública y Privada: ¿Cuál es el giro del negocio?. Santiago: Universidad Alberto Hurtado.

Ball, S. (2015). Estandarización y docencia. (J. Weinstein, Entrevistador).

Ball, S., \& Youdell, D. (2007). Privatización encubierta en la educación pública, infome preliminar, Instituto de Educación, Universidad de Londres. 
La justicia social en el análisis de las políticas educativas: una mirada desde la obra de Stephen Ball

Beech, J., \& Meo, A. (2016). Explorando el uso de herramientas teoricas de Stephen J. Ball en el estudio de las políticas educativas en América Latina. aape-epaa, p.19.

Bourdieu, P., \& Passeron (1996). La reproducción. Elementos para una teoría del sistema de enseñanza. Mexico: Distribuciones Fontamara.

Collins, R. (1989) La sociedad credencialista. En R. Collins, La sociedad credencialista, Ediciones AKAL.

Dye, T. (2008) Understanding Public Policies, 12 th edition. New Jersey: Pearson Prentice Hall.

Fischman, G., Ball, S., \& Gvirtz, S. (2003). Introduction toward a Neoliberal Education? Tension and change in Latin America.

Foucault, M. (2002). Vigilary castigar: nacimiento de la prisión. Buenos Aires: Siglo XXI.

Fraser, N. (2009). Reenquadrando a justiça em um mundo globalizado en LuaNova, São Paulo, No. 77: pp. 11-39.

Fraser, N., \& Honneth, A. (2003). Redistribution or Recognition? A Political - Philosophical Exchange. London: Verso.

Kooiman, J. (2003). Governing as Governance. London: Sage.

Mainardes, J., \& Marcondes, M. (2009). Entrevista com Stephen J. Ball: um diálogo sobre justiça social, pesquisa e política educacional en Educ. Soc., Campinas, Vol. 30, No. 106, pp. 303-318. Recuperado de http://www.cedes.unicamp.br

Pearsons, W. (2007). Políticas Públicas Una introducción a la teoría y la práctica del análisis de políticas educativas. México: Flacso.

<Paradigma> - Revista de Investigación Educativa. Año 24. No. 38 
Puigros, A. (2001). Colección grupos de Trabajo CLACSO. Buenos Aires: CLACSO.

Reimers F. (1999). Educación, pobreza y desigualdad. G. Cordero, Entrevistador. México. 